\title{
Silicon photodiode \& preamplifier characteristic properties under background radiation conditions
}

\author{
V.M. Hodovaniouk, I.V. Doktorovych, V.K. Butenko, V.H. Yuryev, Yu.G. Dobrovolsky \\ Rhythm Optoelectronics Inc., Chernivtsi, Ukraine
}

\begin{abstract}
The investigation results of a silicon photodiode (PD) operation with a preamplifier under background radiation conditions are presented. The preamplifier output signal and its frequency characteristic dependence on the input resistance and capacitance are considered, the influence of the PD radiant-flux characteristic under background radiation and the preamplifier output signal dependence on the background current and intrinsic resistance are investigated. It is shown that the change of the PD parameters under background radiation is similar to the influence of the PD equivalents equivalents of capacitance and resistance.
\end{abstract}

Keywords: photodiode, silicon, preamplifier, radiant-flux characteristic, frequency, background radiation.

Manuscript received 20.12.04; accepted for publication 18.05.05.

\section{Introduction}

As a rule, to investigate light fluxes and fields various photodetectors are used. Experiment results as a whole depend on measurement correctness. This why, the problem of reliability of the results obtained during the photometric measurements always remain as the highpriority one.

In this paper, considered is the case when an optical signal is registered by the photodiode (PD) under background radiation conditions, i.e., the $\mathrm{PD}$ is influenced not only by the light flux under study but also with an additional background flux generated by visible light and other optical sources.

\section{Investigation of the preamplifier operation characteristic properties with the PD equivalent under background radiation}

To a great extent, the PD photoelectric parameters depend on their measurement conditions and modes. Under real conditions, to amplify the signal generated by the PD under the influence of optical radiation a preamplifier is used. It is usually mounted together with the PD or used in the same package as the PD. The PD influence on the preamplifier parameters is rather complex, and the superposition of the background radiation complicates the situation. That's why, to analyze the PD operation with the preamplifier, the PD influence was simulated using the input capacitances and loads.

\subsection{Investigation of output signal and preamplifier frequency characteristics influenced by variable capacitance}

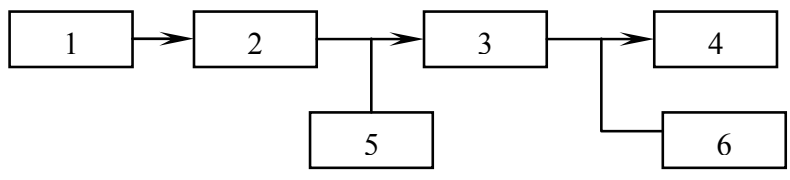

Fig. 1. The block diagram for measurements of the preamplifier assembled frequency characteristics. $1-\mathrm{G} 3-112$ low-frequency generator; 2 - current-holding resistor $R_{r}=$ $=10 \mathrm{MOhm} ; 3-$ amplifier under investigation; $4-\mathrm{B} 3-38$ millivoltmeter; 5 - capacitor (resistor) - PD equivalent; 6 C1-64 oscillograph.

To investigate the preamplifier parameters, there was performed a computer analysis of its frequency characteristic influenced by variable capacitance and resistance connected to the input of the preamplifier. In much the same way the frequency characteristics of the preamplifier assembled were measured. The measurement was made in the range of 2 to $100 \mathrm{~Hz}$ using a test unit, the block diagram of which is shown in Fig. 1.

Equivalent capacitances were chosen within the range 47 to $10,000 \mathrm{pF}$, equivalent resistances - from 100 down to $0.3 \mathrm{kOhm}$.

The results of measuring the frequency characteristic dependence on the input capacitance depicted in Figs 2, 3 . 

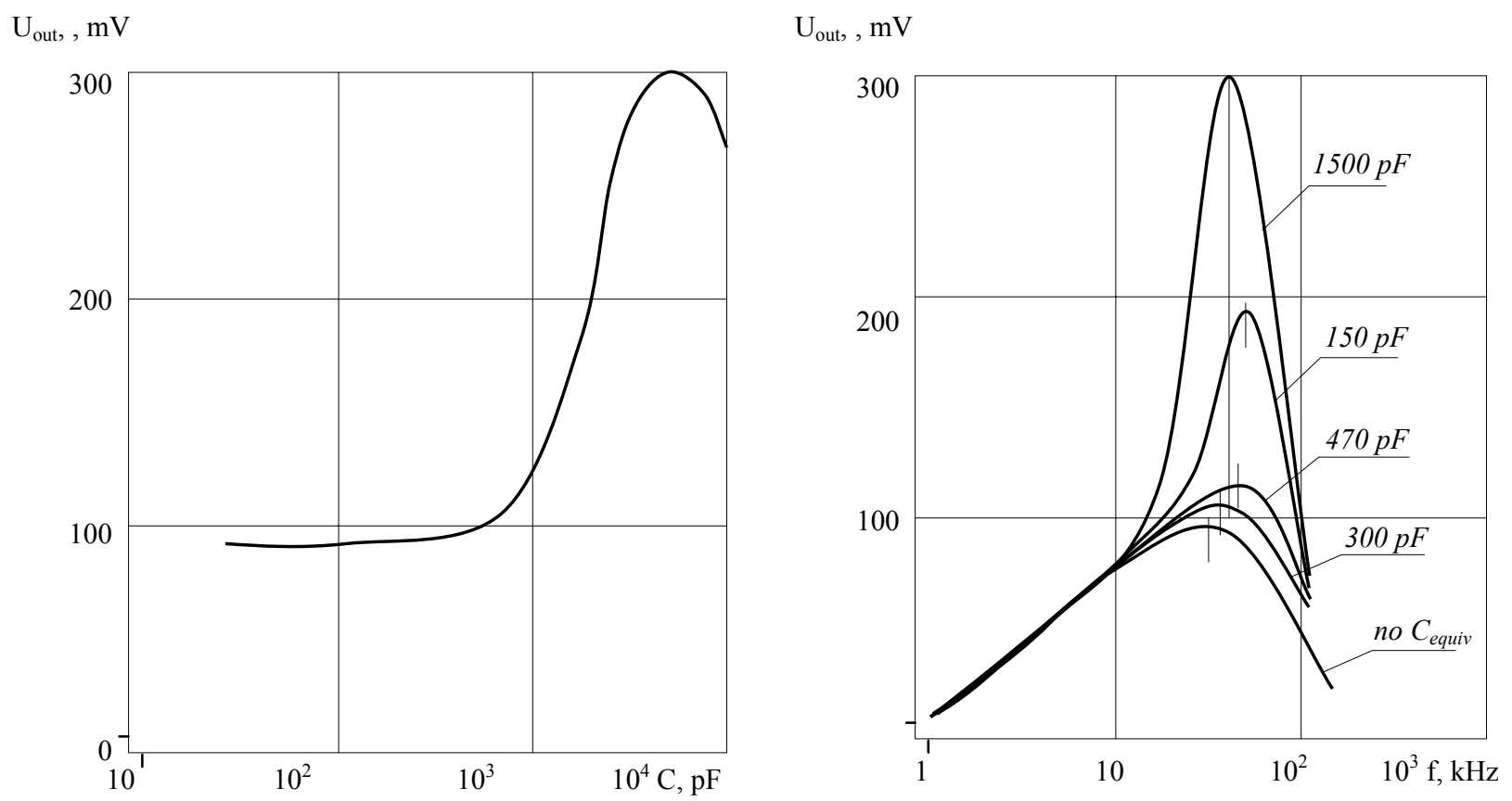

Fig. 2. The preamplifier output signal dependence $\left(f_{m}=\right.$ $=20 \mathrm{kHz}$ ) upon the input capacitance.

Fig. 3. The preamplifier frequency characteristic dependence on the input capacitance.

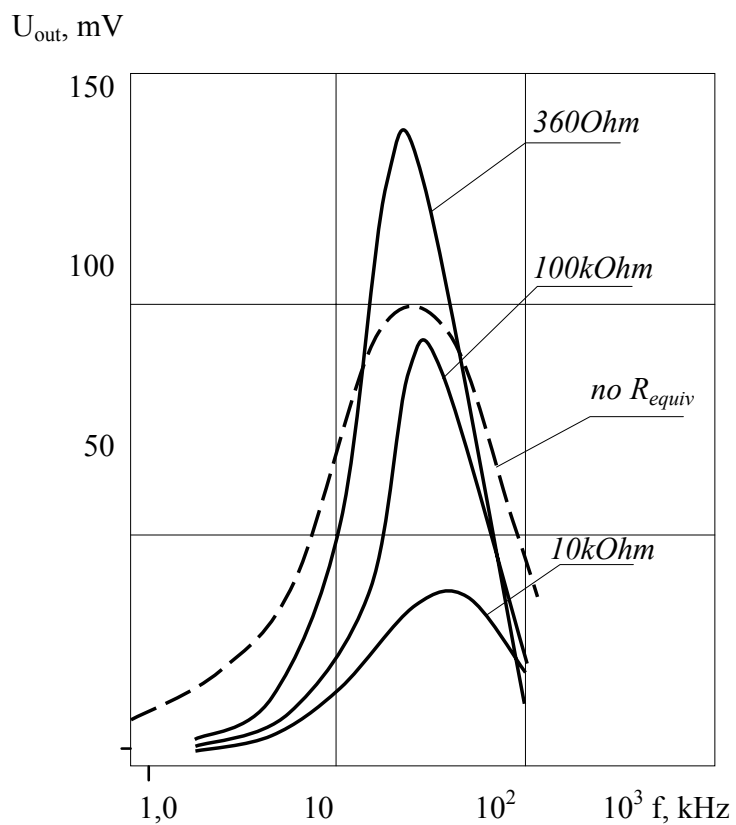

Fig. 5. The preamplifier frequency characteristic as a function

Fig. 4. The preamplifier output signal $\left(f_{m}=20 \mathrm{kHz}\right)$ of the input resistance. 
2.2. Investigation of the output signal and the preamplifier frequency characteristics influenced by variable resistance

Similarly, investigations of the preamplifier amplitude-frequency characteristics under input resistance variation were carried out. The measurement results of the frequency characteristic dependence on the input resistance are shown in Figs 4, 5.

After analyzing the measured preamplifier frequency characteristics, one can draw a conclusion that these characteristics are similar to the simulated ones in their amplitude and shape.

\section{Investigation of the preamplifier operation characteristic properties with a real PD under background radiation conditions}

As far as the photoelectric parameters of a real PD to a great extent depend on their measurement conditions and mode, in real conditions, its influence on the preamplifier parameters is more complex, and it is sufficiently difficult to make a real PD equivalent.

\subsection{Investigation of the PD current monochromatic responsivity under background radiation conditions}

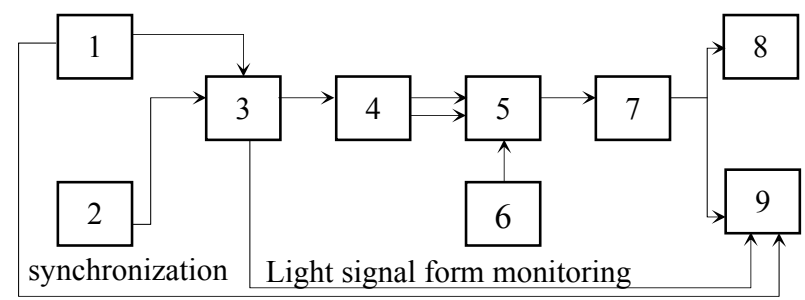

Fig. 6. The test unit block diagram: $1-\mathrm{G} 3-112$ low-frequency generator; 2 - B5-30 emitter power supply unit; 3 - contact device; 4 - LED of operating wavelength; 5 - PD; 6 - PD B5-43 power supply unit; 7 - transducer; 8 - B3-38 millivoltmeter; 9 - C1-64 oscillograph.

Note. As the converter 7 we used the following: 1 - U2-8 (load in the PD circuit $R_{1}=1 \mathrm{kOhm}$ and $R_{2}=10 \mathrm{kOhm}$ ); 2 - currentvoltage transducer (CVT) developed by Rhythm Optoelectronics (conversion ratio of $K_{1}=10^{3} ; \quad K_{2}=10^{4}$; $K_{3}=10^{5} ; K_{4}=10^{6}$, and $\left.K_{5}=10^{7} \mathrm{~V} / \mathrm{A}\right)$.

To provide correct measurements of the PD current monochromatic responsivity under background radiation conditions, Rhythm Optoelectronics Inc. used a specially developed test unit, the block diagram of which is presented in Fig. 6.

To derive the emitter frequency characteristic from the measurement results, its frequency characteristic was determined. For this purpose, there were used a lowresponse-time silicon reference $\operatorname{PD}\left(U_{\text {op }}=200 \mathrm{~V}\right)$ and the CVT with the frequency characteristic not less than $200 \mathrm{kHz}$ at $K=10^{4} \mathrm{~V} / \mathrm{A}$. As a result of the measurement (see Table), it was ascertained that the radiation flux level is frequency-independent (in the

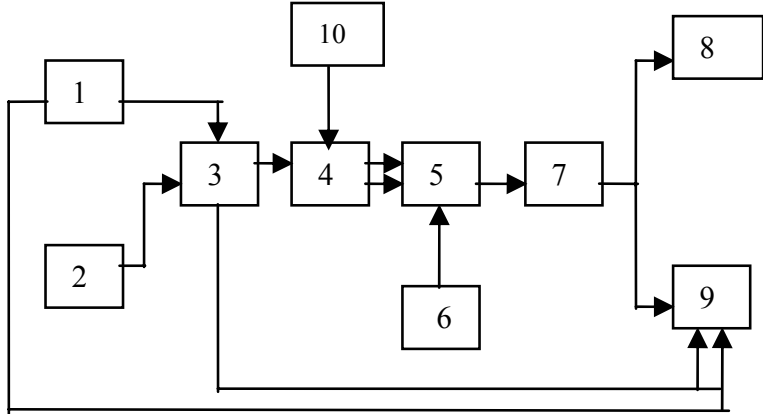

Fig. 7. 1 - G3-112 low-frequency generator; 2 - B5-30 emitter supply unit; 3 - ADB7.5490 contact device; 4 - LED of $\lambda_{\max }=1.06 \mu \mathrm{m} ; 5-$ PD under investigation; $6-\mathrm{B} 5-43$ PD power supply unit; 7 - preamplifier $(R=510 \mathrm{kOhm}) ; 8$ V3-38 voltmeter; 9 - C1-64 oscillograph; 10 - B5-44 power supply unit of a background radiation source.

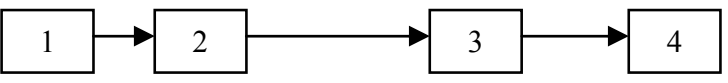

Fig. 8. 1 - B5-44 emitter power supply unit; 2 - background radiation source - the AL107 LEDs array; 3 - PD under investigation; 4 - M2015 voltammeter.

measuring circuit presented) up to $50 \mathrm{kHz}$, and, thus, the emitter does not influence on the measurement results.

As a result of these investigations, the absolute values of the current monochromatic responsivity appeared to be reliable, being measured using the above circuit with the U2-8 transducer and the load $1 \mathrm{kOhm}$. In measurements according to other techniques, a great mistake appears because of the influence of PD parameters on the transducer and the PD amplitudefrequency characteristics.

3.2. Investigation of the PD parameters influence on the preamplifier output signal under background radiation conditions

To investigate the influence of the PD parameters on the preamplifier output under background radiation conditions, an emitter providing the PD radiation with operating and background fluxes was produced. The operating radiation level provided the measurement of the PD parameters on the linear section of the photocurrent-radiant flux characteristic, and remained unchanged during the measurements.

The results of measuring the emitter amplitude-frequency characteristics.

\begin{tabular}{|l|c|c|c|c|c|}
\hline$f, \mathrm{kHz}$ & 0.1 & 1.0 & 10 & 20 & 50 \\
\hline $\begin{array}{l}\text { Light flux, } \\
\text { rel. units }\end{array}$ & 1.0 & 1.0 & 1.0 & 1.0 & 1.0 \\
\hline
\end{tabular}




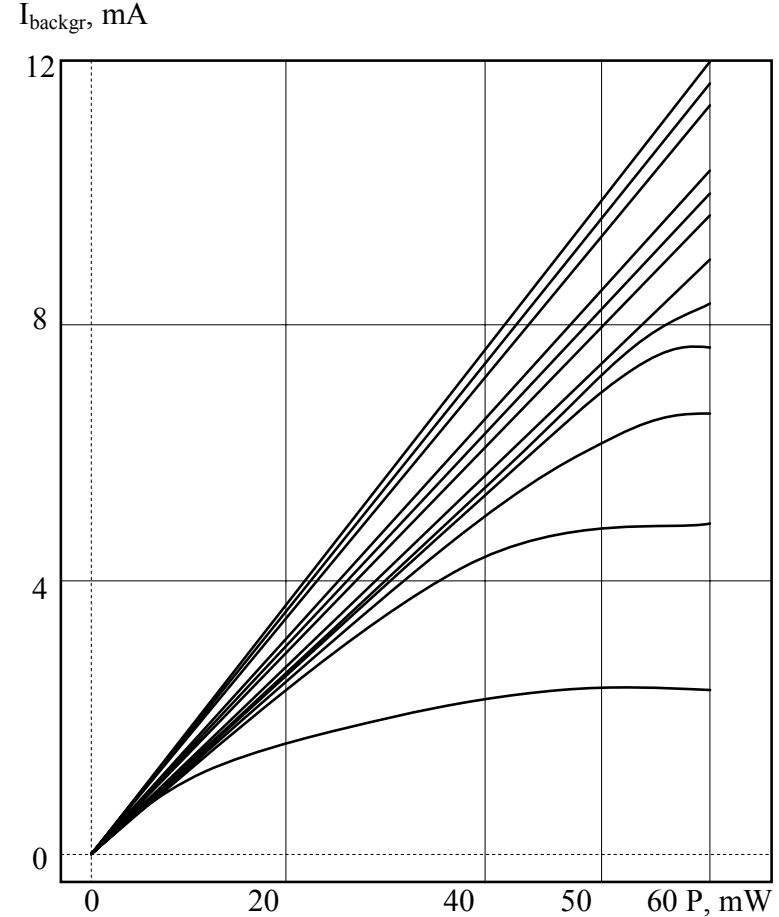

Fig. 9. The radiant-flux characteristic of various uniform PDs

The measurement of the preamplifier output signal under background radiation was carried out using the simulating model unit, the structural model of which is shown in Fig. 7.

The measurement was conducted in the following way: after illumination of the PD by the operation flux of the optical radiation, the signal at the input of the preamplifier was measured under no-background radiation conditions. Then, the background radiation was moothly increased up to the value at which the signal disappeared. The level at which the signal disappeared appeared to be different. It is different even for a PD with the same responsivity values. It can be explained by the fact that the dynamic range, and the compensation circuit of the preamplifier background signal balances out a certain level of the background signal, if different PDs is not the same.

To measure the dynamic range of the $\mathrm{PD}$, the simulating model unit was used. Its structural model is shown in Fig. 8.

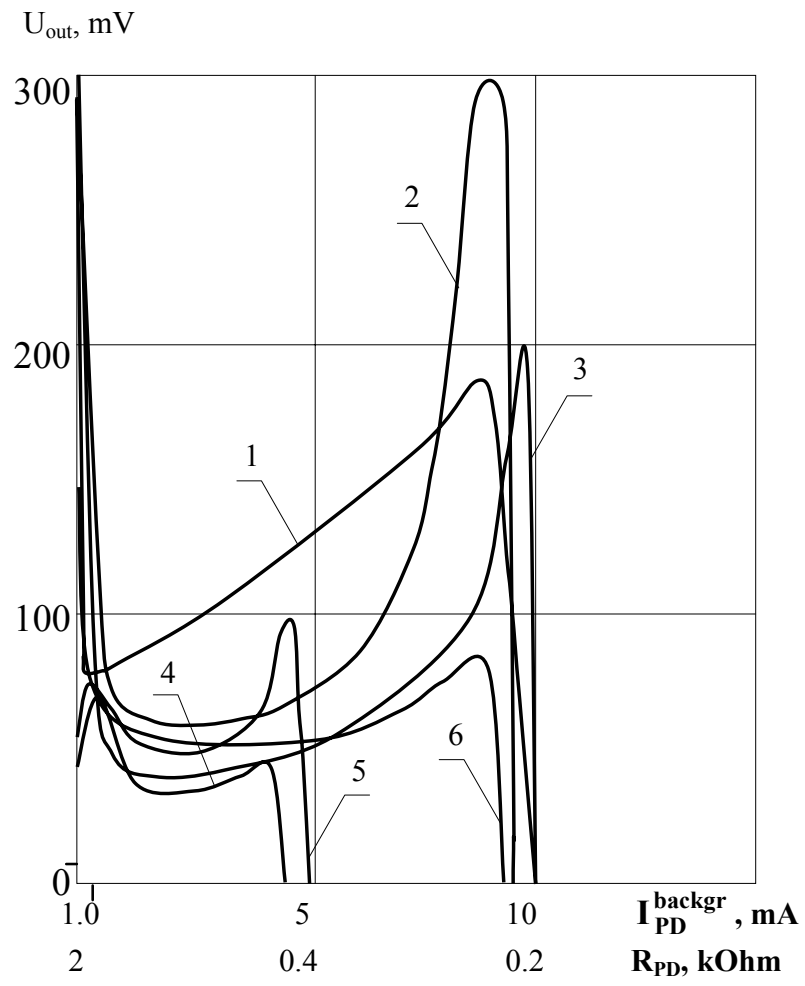

Fig. 10. The preamplifier output signal as a function of the input background current $\left(I_{\text {backgr }}\right)$ and intrinsic resistance $\left(R_{\mathrm{PD}}\right)$ : $1-U_{\text {out }}=f\left(R_{\text {equiv }}\right) ; 2-6-$ dependences for PDs of different radiant-flux characteristic linearity levels (N 2 of linear characteristic up to $12 \mathrm{~mA}, \mathrm{~N} 4-$ up to $1 \mathrm{~mA}$ ).

The measurement results of PD dynamic range and the preamplifier output signal under background radiation are presented in Figs 9 and 10, accordingly.

\section{Conclusion}

Thus, our investigation of the preamplifier output signal under background radiation conditions has shown that the change of the PD parameters under the influence of the background radiation is analogous to the influence of the PD equivalents - the equivalents in capacitance and resistance. That's why, to apply the PD in corresponding equipment it is necessary to take into account its dynamic range, and the frequency characteristics of the preamplifier that should be expanded to the values at which the background radiation will be able to take out of the limits of the system operating frequency. 